\title{
Paget's Disease of Bone
}

\author{
Jaime Paulos
}

\section{Abstract}

Paget's disease of bone is very common in older people, secondary to an altered remodeling, deforming bone structure. X-rays are characteristic. Most cases are asymptomatic, but can attain pathological fractures and long term infrequent cases of secondary sarcomas.

\section{Keywords}

Paget'S disease $\cdot$ osteitis deformans

Paget's disease (osteitis deformans) is a skeletal disease that results from an altered bone remodeling with accelerated resorption in some areas and abundant new bone formation in others [1-3]. The most common form of presentation is poorly localized bone pain and additionally it can present itself with pathologic bone fractures. The pelvis, spine, skull, and proximal long bones are most frequently affected. The $\mathrm{X}$-ray is characteristic $[4,5]$. Serum alkaline phosphatase is elevated because of the high bone turnover rate. It is a common finding in older people. It can be mono or poliostotic.The mixed (middle) phase of Paget's disease combine characteristics of both the lytic (initial) and the blastic (late) phases. Most patients present in the mixed phase when decreased osteoclastic activity and increased osteoblastic activity are encountered. Symptomatic cases can be treated with bisphosphonates. The most advanced form is the malignant transformation to a secondary sarcoma (osteosarcoma) [2] that has a poor prognosis.

\section{References}

1. Kaplan FS, Singer FR. Paget's disease of bone: pathophysiology, diagnosis, and management. J Am Acad Orthop Surg. 1995;3(6): 336-44.

2. Wick MR, Siegel GP, McLeod RA. Sarcomas of bone complicating osteitis deformans (Paget's disease): fifty years experience. Am Pathol J Surg. 1981; 5:47-59.

3. Resnick D. Paget's disease, diagnosis of bone and joint disorders. 4th ed. Philadelphia, PA: Saunders; 2002. p. 1947-2000.

4. Whitehouse RW. Paget's disease of bone. Semin Musculoskelet Radiol. 2002;6:313-22.

5. Mirra JM, Brien EW, Tehranzadeh J. Paget's disease of bone: review with emphasis on radiologic features-part I. Skeletal Radiol. 1995;24:173-84. 